\title{
How sticky is the chaos/order boundary?
}

\author{
Carl P. Dettmann
}

\begin{abstract}
In dynamical systems with divided phase space, the vicinity of the boundary between regular and chaotic regions is often "sticky," that is, trapping orbits from the chaotic region for long times. Here, we investigate the stickiness in the simplest mushroom billiard, which has a smooth such boundary, but surprisingly subtle behaviour. As a measure of stickiness, we investigate $P(t)$, the probability of remaining in the mushroom cap for at least time $t$ given uniform initial conditions in the chaotic part of the cap. The stickiness is sensitively dependent on the radius of the stem $r$ via the Diophantine properties of $\rho=(2 / \pi) \arccos r$. Almost all $\rho$ give rise to families of marginally unstable periodic orbits (MUPOs) where $P(t) \sim C / t$, dominating the stickiness of the boundary. Here we consider the case where $\rho$ is MUPO-free and has continued fraction expansion with bounded partial quotients. We show that $t^{2} P(t)$ is bounded, varying infinitely often between values whose ratio is at least $32 / 27$. When $\rho$ has an eventually periodic continued fraction expansion, that is, a quadratic irrational, $t^{2} P(t)$ converges to a log-periodic function. In general, we expect less regular behaviour, with upper and lower exponents lying between 1 and 2. The results may shed light on the parameter dependence of boundary stickiness in annular billiards and generic area preserving maps.
\end{abstract}

\section{Introduction}

Understanding Hamiltonian dynamics with mixed regular and chaotic phase space remains one of the most important and intractable problems in dynamical systems, with many open questions. Much of the difficulty of such systems is that often (and probably "typically" in many senses) the boundary between regular and chaotic regions (however defined) is fractal. This is true even for well studied and visualised classes of systems such as two dimensional billiards, in which a point particle (of unit mass and speed, without loss of generality) moves uniformly, making mirror-like reflections with the boundary of a domain $D \subset \mathbb{R}^{2}[\mathbf{1 1}, \mathbf{2 2}$. Dynamics in any billiard has one natural, "equilibrium" invariant measure: For the flow (continuous time) dynamics it is uniform (that is, proportional to Lebesgue) in position in the domain and in the direction of motion. For the map (from one collision to the next) it is uniform in arc length and the tangential component of velocity. 

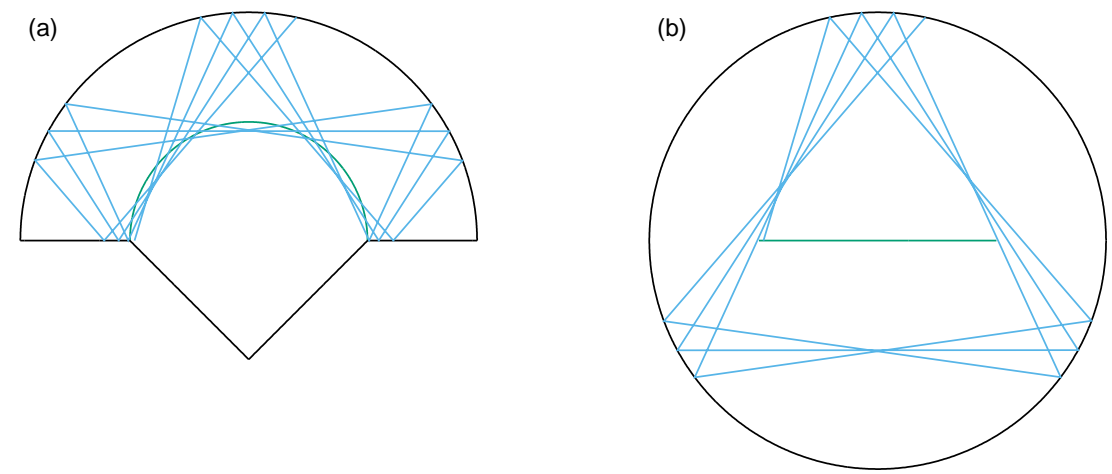

FiguRE 1. (a) The mushroom geometry; the radius of the cap is 1 and that of the stem is $r \in(0,1)$. (b) Reduction of dynamics in the cap to a circular billiard, by reflecting across the $x$-axis. The stem becomes a slit-like hole of size $2 r$ in the interior. An orbit with rotation number close to $1 / 3$ is shown in each case.

Mushroom billiards were introduced by Bunimovich, as the first examples of billiards with sharply divided regular and chaotic phase space [8]. The simplest mushroom consists of a semicircular cap (of unit radius, without loss of generality) and rectangular or triangular symmetrically placed stem of radius $r \in(0,1)$; see Fig. 1. Orbits with angular momentum (that is, distance of closest approach to the centre) $l>r$ in the cap cannot reach the stem, and so form a regular component with rotation number $\lambda / 2$ where

$$
\lambda=\frac{2}{\pi} \arccos l
$$

due to the integrability of the circle. This region corresponds to $\lambda<\rho$ where

$$
\rho=\frac{2}{\pi} \arccos r
$$

The factor 2 in these definitions is needed to simplify the Diophantine approximation conditions; see Ref. 15 and below. The set of orbits that enter the stem forms the chaotic component, by an application of the defocusing mechanism under which a large class of billiards with focusing boundary components (including the well known stadium) can be shown to exhibit chaos 7 . In fact, the mushroom limits to both the regular circle and ergodic semi-stadium in the limit of small and large stem radius, respectively. More complicated mushrooms may involve semiellipses and/or be constructed to have an arbitrary number of regular and chaotic components $[\mathbf{8}, \mathbf{1 0}]$. 
Later, it was observed that even in mushroom billiards, the simple structure of the phase space may be complicated by the presence of marginally unstable periodic orbits (MUPOs) embedded in the chaotic region [2]. As is common in the literature, we use the term MUPO to denote the whole continuous family of periodic orbits with a given rational rotation number. These are any periodic orbit $(\lambda \in \mathbb{Q})$ restricted to cap of the mushroom (hence their marginal, ie parabolic nature) but have $\lambda \geq \rho$ (hence located in the chaotic region or its boundary). When $\lambda$ is perturbed, the orbit precesses for a long time but eventually falls into the stem and into the main body of the chaotic region. MUPOs are thus responsible for the phenomenon of stickiness, the phenomenon in which chaotic orbits spend long periods in quasi-regular behaviour. If the mushroom stem has no periodic orbits entirely contained within it, such as the triangular stem in Fig. 1, any MUPOs in the cap are the main source of stickiness.

Note that there are a great variety of examples of stickiness in billiards, as discussed recently [10. "Internal" stickiness is where there is no island of stability, such as the original stadium billiard, or where stickiness is due to MUPOs completely contained in the chaotic sea, such as the mushroom MUPOs above. In contrast, "external" stickiness is related to the boundary between chaotic and regular regions. Ref. 10 gives a number of examples of external stickiness, arguing that where the orbits in the regular region are parabolic (as in mushrooms with circular caps) the island is typically not sticky, and giving as a counterexample the case where the boundary corresponds to rational rotation number (ie a MUPO). While MUPOs lead to stickiness located in the chaotic sea (internal stickiness), the nature of mixed phase space also requires an understanding of the stickiness of the boundary between the regular and chaotic regions (external stickiness).

Here we characterise stickiness in terms of an open billiard. If initial conditions are distributed with respect to the equilibrium invariant measure of the flow, restricted to the cap of a mushroom and to the chaotic region $\lambda>\rho$, and the stem is replaced by a hole, we can consider the survival probability $P(t)$, that the particle has not escaped by time $t$. A MUPO leads to a contribution to $P(t)$ proportional to $1 / t$ as $t \rightarrow \infty$ 15. That work also gave a number of results (discussed below) about MUPOs in mushrooms, including characterising the set of $r$ for which there are no MUPOs, including an explicit example, namely $r=\cos \left(\frac{5+\sqrt{2}}{23} \pi\right) \approx 0.640134$.

This paper is concerned with the stickiness of the boundary, which is weaker than stickiness due to MUPOs. It was conjectured in Ref. [15] that when there are no MUPOs the boundary would contribute $C / t^{2}$. Subsequently Alastair Robertson's undergraduate project [18] with more careful numerics for the above explicit $r$, suggested this form of decay, but with $C$ bounded but not convergent. This calculation is replicated with a larger sample size of $10^{11}$ in Fig.2.

Here we find that not only the existence of MUPOs has an intricate parameter dependence, but also the boundary stickiness. In particular, the stickiness depends sensitively on the Diophantine properties of $\rho$. In this paper we give results when the partial quotients $a_{n}$ in the continued fraction expansion are bounded:

TheOREM 1.1. Mushrooms with bounded partial quotients. Consider a MUPOfree mushroom for which $\rho$ has bounded partial quotients. Then

$$
\frac{\limsup t^{2} P(t)}{\liminf t^{2} P(t)} \geq \frac{32}{27} \approx 1.185 \ldots
$$




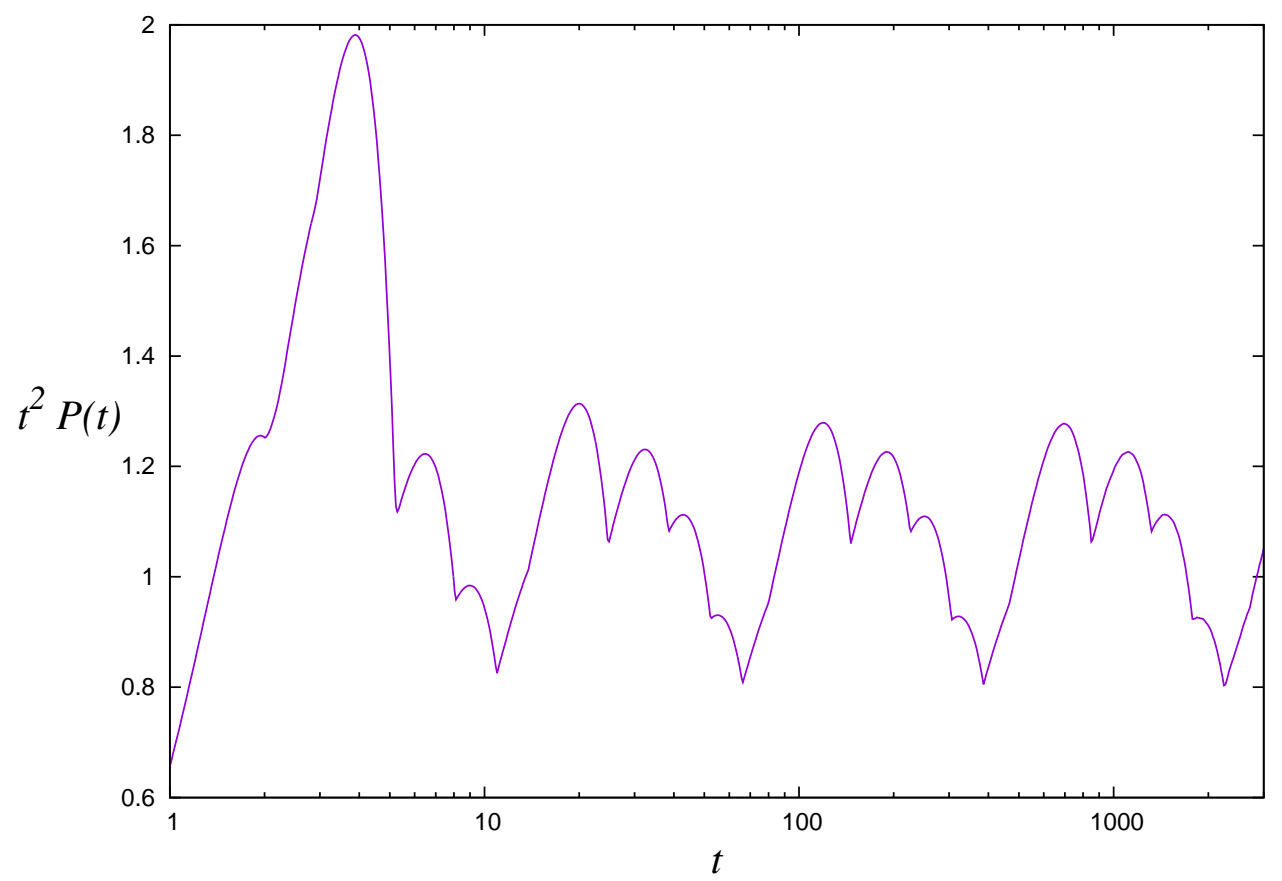

Figure 2. In the MUPO-free case $r=\cos \left(\frac{5+\sqrt{2}}{23} \pi\right) \approx 0.640134$ the survival probability limits to a log-periodic function divided by $t^{2}$, as shown by Thm. 1.2

as $t \rightarrow \infty$, and in particular both limits are positive and finite.

The main idea of the proof is to show that the graph of $P(t)$ is approximately piecewise linear with the ratio between nonsmooth abscissas at least the golden ratio infinitely often, and that a linear piece with this ratio has $t^{2} P(t)$ varying by at least a factor of $32 / 27$. In the special case where the partial quotients are not only bounded but eventually periodic we have

THEOREM 1.2. Mushrooms with eventually periodic partial quotients. Consider a MUPO-free mushroom for which $\rho$ has eventually periodic partial quotients. Then there is a constant $\beta>1$ so that the limit taken over integers $m$,

$$
\lim _{m \rightarrow \infty} \beta^{2 m} t^{2} P\left(\beta^{m} t\right)
$$

converges for all $t>0$.

The even partial quotients of $\rho$ must be bounded for the mushroom to be MUPO-free, from previous studies (see Sec. 2 below). However there is no such constraint on the odd partial quotients; typical MUPO-free mushrooms (if a measure on the set of MUPO-free $\rho$ can be naturally defined) would be expected to have unbounded odd $a_{n}$, and indeed these values could grow rapidly. It would be interesting to extend the methods developed here to these cases. For now, we present only the example in Fig. 3, also with a sample size of $10^{11}$. 


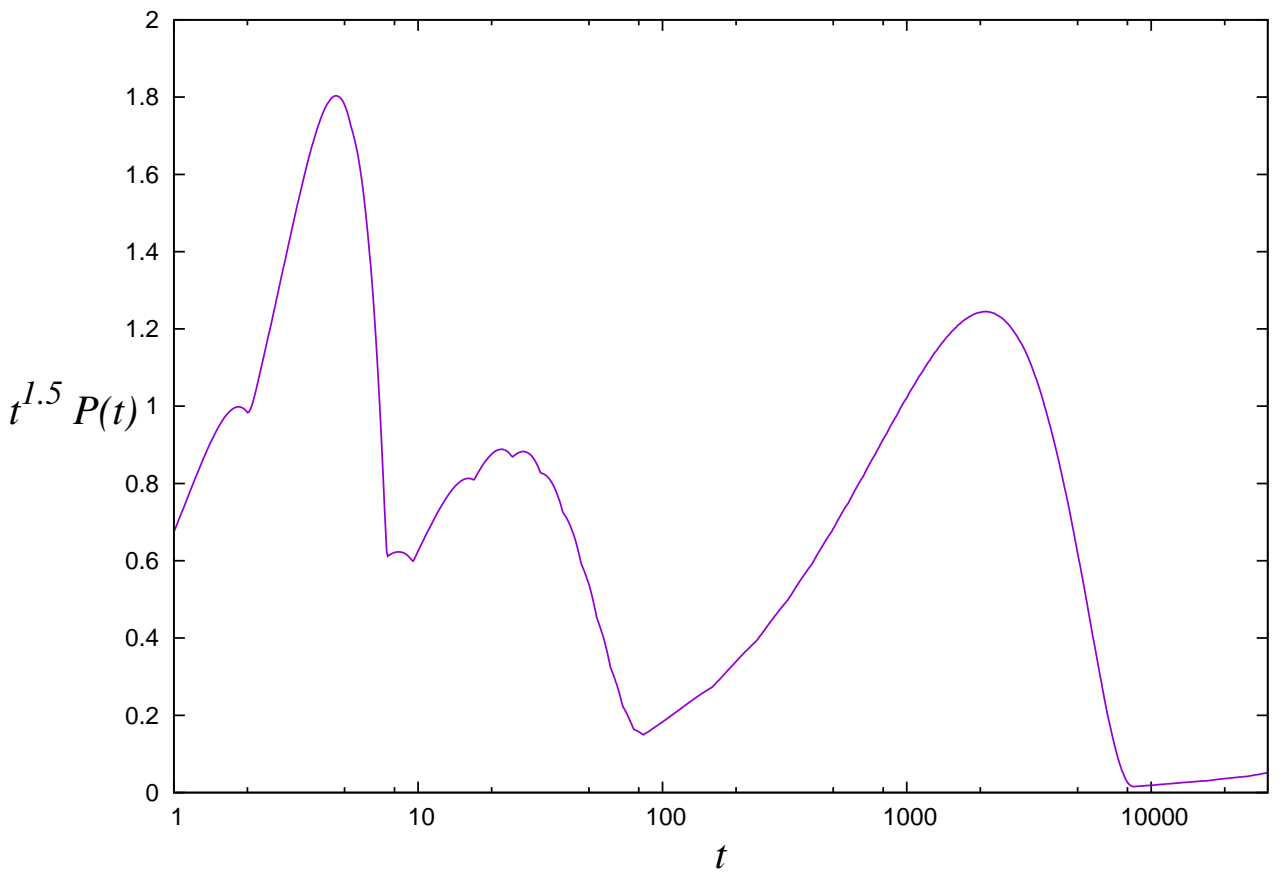

Figure 3. A MUPO-free example constructed from the wellapproximable $\rho=[0 ; 1,3,10,1,100,1,1000, \ldots]$ which gives $r \approx 0.374600$. An exponent for the survival probability $-\lim _{t \rightarrow \infty} \frac{\ln P(t)}{\ln t}$ does not appear to exist.

It appears that such behaviour may be arbitrarily close to that of MUPOs (see also Thm 1 in Ref. 14 for a similar result in open chaotic maps):

CONJECTURE 1.3. Liouville mushrooms. For sufficiently well-approximable MUPO-free $\rho$,

$$
\limsup _{t \rightarrow \infty} \frac{-\ln P(t)}{\ln t}=1
$$

There are some other systems to which the present results could apply. Mushrooms are of particular interest in quantum mechanics, in which the classical dynamics approximates the small wavelength limit of the Schrödinger equation with (typically) Dirichlet conditions on the boundary. Here, wave functions corresponding to different components of phase space, and tunnelling rates between these components, can be observed numerically and experimentally [5, 6, 16, 17, 25]. Thus, it would be of interest to know in what way the amount of classical stickiness affects the quantum mechanical properties such as the energy level spacing.

A closely related class of billiards, also used in experiments, is that of the annulus, a circle with a circular scatterer [13. In this case orbits with sufficiently large angular momentum cannot reach the scatterer and form a regular component of phase space. Orbits reaching an off-centre scatterer may be chaotic or belong 
to elliptic islands. It would be interesting to study boundary stickiness in both classical and quantum annuli.

Finally, we return to generic area preserving maps. Given the subtleties for the case of sharply divided phase space, it is unsurprising that the generic mixed boundary has eluded detailed understanding for so long. There are similarities between the mushroom and generic cases, for example the importance of one-sided Diophantine approximation; see Ref. 4 for a recent study focusing on the Hénon map, and references. There also, the rotation number of the invariant boundary circle depends intricately on the control parameter. This suggests that there may be an exceptional set of parameters in which the non-integer universal decay exponent as conjectured in Ref. $\mathbf{1 2}$ does not hold.

The outline of this paper is as follows: Section 2 summarises relevant previous results, section 3 develops the theory, culminating in proofs of the two theorems in section 4, with more technical lemmas and their proofs relegated to section 5 . Sec. 3.3 demonstrates the possibility of an incipient MUPO not leading to stickiness, but contains a further conjecture that they are not found in otherwise MUPO-free mushrooms.

\section{Acknowledgements}

This paper is dedicated to the memory of Nikolai Chernov, whose life continues to inspire and enlighten all who play mathematical billiards. Thanks to the organisers of his memorial conference in Birmingham AL, May 2015 for their kind hospitality. The author is grateful to Alastair Robertson, whose undergraduate project report $1 \mathbf{1 8}$ included an early version of Fig. 2, providing the impetus for this work; also to Leonid Bunimovich and Orestis Georgiou for helpful discussions and to user O.L. on math.stackexchange.org for assistance with the final integral in Eq. (3.15). This work was supported by the EPSRC [grant number EP/N002458/1]. Data underlying this work (used to illustrate, not assist, the proofs) is available from the University of Bristol data repository at https://dx.doi.org/10.5523/bris.ujws3ienvh8q1arcsm7c4q7ew

\section{Previous results}

This section gives a brief summary of previous results on MUPOs in mushrooms, or lack thereof. In mushrooms with circular caps, each MUPO (defined by coprime integers $1 \leq A<B)$ exists for a fixed interval of stem radii $[\mathbf{2}$, $\mathbf{1 5}$.

$$
\cos \left(\frac{\pi}{2} \frac{A}{B}\right) \leq r<\frac{\cos \left(\frac{\pi}{2} \frac{A}{B}\right)}{\cos \left(\frac{\pi}{2} \frac{1}{B}\right)}
$$

For a fixed mushroom, the amount and type of stickiness depends on what MUPOs exist. This investigation was initiated by Altmann and coauthors 1, 2, 3, who showed that for both mushroom and annular billiards, the MUPOs are related to the Diophantine properties of the relevant parameter, here $\rho$. MUPOs appear when there is sufficiently good one-sided approximation of the component boundary by orbits with rational rotation numbers, for example corresponding to unbounded even partial quotients in the continued fraction expansion of $\rho$. Such behaviour is typical, so that a full measure of parameters have infinitely many MUPOs. Ratio-

nal $\rho$ yield a positive finite number of MUPOs, including the one exactly on the 
boundary. Tsugawa and Aizawa have recently studied the Fibonacci case, that is, the most extreme badly approximable $\rho[\mathbf{2 3}$.

Further results were provided by Dettmann and Georgiou [15], who showed that there were parameter values with no MUPOs, gave a method for finding them, and the explicit example $r=\cos \left(\frac{5+\sqrt{2}}{23} \pi\right) \approx 0.640134$. The idea here is that quadratic irrationals have periodic (and in particular bounded) continued fraction expansions; a finite number of other conditions needed to be checked. Periodicity is not necessary; any value with bounded even partial quotients and sufficiently small stem, specifically $a_{\max }=\sup a_{2 n}$ and

$$
r<\frac{1}{\sqrt{\pi^{2}\left(a_{\max }+2\right)^{2}+1}}
$$

will have finitely many MUPOs, and usually zero (can be proved for specific $r$ by checking a finite number of conditions) as in the example in Fig. 3.

Ref. 15. also showed that the supremum of MUPO-free radii is $\frac{1}{\sqrt{2}} \approx 0.707107$, and that the supremum of finitely many MUPOs for irrational rotation number is $\frac{4}{\sqrt{16+\pi^{2}}} \approx 0.786439$. Bunimovich has recently provided an alternative characterisation of the MUPO-free parameter set, including a number of theorems, bounds on MUPO numbers and method for finding MUPO-free mushrooms [9].

\section{Development of the theory}

3.1. From the mushroom to circle rotations. The mushroom geometry we consider is shown on the left in Fig. 1. The cap has radius 1 while the stem has radius $r \in(0,1)$ and has a polygonal geometry that contains no periodic orbits entirely within it. An example with periodic orbits would be a rectangle, with horizontal period two orbits. Any such periodic orbits would be MUPOs and lead to stickiness in the chaotic region, since all orbits of polygonal billiards are parabolic. Apart from the no-MUPO constraint, the stem geometry is arbitrary, and not relevant to the analysis below. Note that many other mushroom geometries can be constructed with different and interesting properties $\mathbf{1 0}$.

Using the usual reflection trick, any orbit which hits only the cap is equivalent to an orbit in the circle obtained by reflecting the cap in its straight sides (right of Fig. 11. The stem then becomes a slit in the interior of the circle. A MUPO is any periodic orbit which remains in the cap, but which intersects the circle of radius $r$ shown on the left of Fig. 1. A MUPO orbit may be rotated around the circle (showing that these come in continuous families of orbits), however a small perturbation which changes its rotation number causes it to precess, and eventually reach the stem, as demonstrated for the orbit shown, which is near a period three MUPO.

We then use the 2-fold rotational symmetry of the circle and hole, and identify opposite points. The collision map then corresponds to a circle rotation

$$
\Phi(x)=\{x+\lambda\}
$$

where $\pi x$ is arc length and \{\} denotes fractional part (ie mod 1). The slit corresponds to a hole (single due to the symmetry reduction) in the $x$ dynamics of size

$$
h=\frac{2}{\pi} \arccos \frac{l}{r}=\sqrt{\frac{4}{\pi} \tan \frac{\pi \rho}{2}}(\lambda-\rho)^{1 / 2}+\mathcal{O}(\lambda-\rho)^{3 / 2}
$$


where (recalling Eqs. 1.1, 1.2

$$
\begin{aligned}
& l=\cos \frac{\pi \lambda}{2} \\
& r=\cos \frac{\pi \rho}{2}
\end{aligned}
$$

We consider only the chaotic part of the mushroom cap $\rho<\lambda<1$. In continuous time, the collisions occur at intervals

$$
\tau_{\lambda}=2 \sqrt{1-l^{2}}=2 \sin \frac{\pi \lambda}{2}
$$

Here, we are interested in mushrooms without MUPOs. As shown in Refs. 1, 15, 9 this corresponds to a set of $r$ with zero Lebesgue measure with a supremum at $r=1 / \sqrt{2}$, described using the Diophantine properties of $\rho$.

3.2. Continued fractions and the three gap theorem. Dynamics in a circle is described by the three gap theorem $\left[\mathbf{2 0}, \mathbf{2 1}, \mathbf{2 4}\right.$; that is, the set $\left\{\Phi^{i}(x)\right\}_{i=0 \ldots N-1}$ has gaps between adjacent points on the circle of at most three different sizes, which are determined by $N$ and the continued fraction expansion of $\lambda$. We follow the first chapter of Ref. [19, extending the notation to the "semiconvergents": Given a standard continued fraction for $0<\lambda=a_{0}+1 /\left(a_{1}+1 /\left(a_{2}+\ldots\right)\right)=\left[a_{0} ; a_{1} a_{2} \ldots\right]$ containing partial quotients $a_{j} \in \mathbb{Z}$, with $a_{0} \geq 0$ (here $a_{0}=0$ ) and all other $a_{j} \geq 1$ we define $B_{-1}=0, A_{-1}=B_{0}=1, A_{0}=a_{0}, A_{k}=A_{k, a_{k}}$ and $B_{k}=B_{k, a_{k}}$ with

$$
\begin{aligned}
& A_{k, i}=i A_{k-1}+A_{k-2} \\
& B_{k, i}=i B_{k-1}+B_{k-2}
\end{aligned}
$$

for $k \geq 1$ and $1 \leq i \leq a_{k}$. Then $A_{k} / B_{k}$ are the convergents (closest approximants) to $\lambda$ and $A_{k, i} / B_{k, i}=\left[a_{0} ; a_{1} a_{2} \ldots a_{k-1} i\right]$ are semiconvergents. The complete quotients are defined as $\zeta_{k}=\left[a_{k} ; a_{k+1} a_{k+2} \ldots\right]$ and hence lie between $a_{k}$ and $a_{k}+1$. In terms of these we have for any $k \geq 0$,

$$
\lambda=\frac{A_{k} \zeta_{k+1}+A_{k-1}}{B_{k} \zeta_{k+1}+B_{k-1}}
$$

The differences are $D_{k}=D_{k, a_{k}}$ with

$$
\begin{aligned}
D_{k, i} & =B_{k, i} \lambda-A_{k, i} \\
& =i D_{k-1}+D_{k-2} \\
& =(-1)^{k} \frac{\left(a_{k}-i\right) \zeta_{k+1}+1}{B_{k} \zeta_{k+1}+B_{k-1}}
\end{aligned}
$$

using the relation $A_{k} B_{k-1}-A_{k-1} B_{k}=(-1)^{k+1}$. Thus we can determine the sign and bound the magnitude of the differences.

These differences are plotted in Fig. 4, a union of straight line segments labelled by coprime postive integers $(A, B)$. Each has $x$-intercept $A / B$ and gradient $B$. The final partial quotient of a continued fraction expansion of $A / B$ is $a_{k}=i$. Each straight line segment corresponds to two $(k, i)$, even $k$ for $D>0$, odd $k$ for $D<0$, and $i=1$ for the larger $k$, corresponding to the two continued fraction representations of the rational $A / B$. Each segment extends to values of $\lambda$ equal to the truncated continued fractions (Stern-Brocot parents) of $A / B$.

In our notation, the three gap theorem says that we need to find the largest $B_{k, i} \leq N$. Then there are $N-B_{k-1}$ gaps of size $\left|D_{k-1}\right|, N-B_{k, i}$ gaps of size 


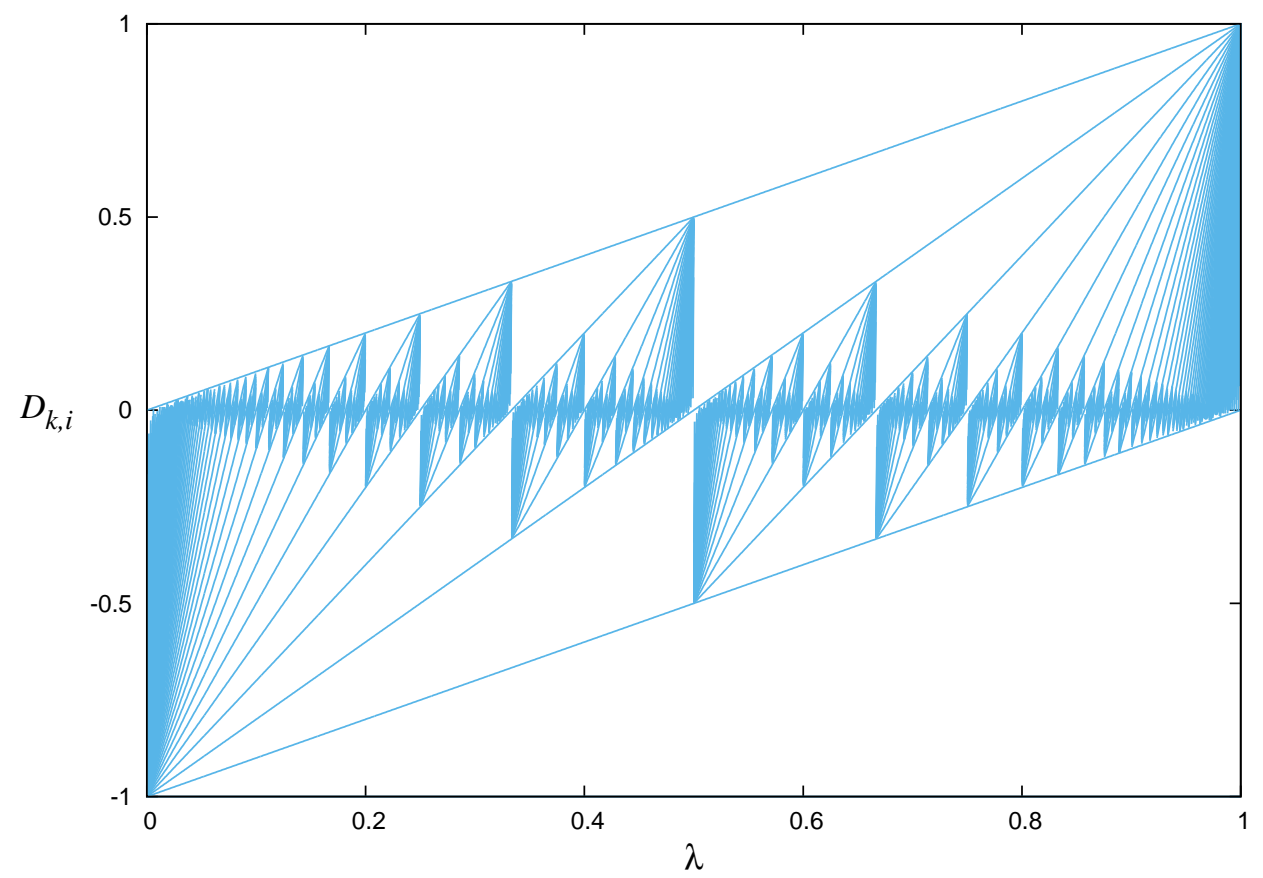

Figure 4 . The differences $D_{k, i}$ as a function of $\lambda$.

$\left|D_{k, i}\right|$ and the remaining $B_{k-1}+B_{k, i}-N$ (possibly zero) gaps are the sum of the previous two sizes, which comes to $\left|D_{k, i-1}\right|\left(=\left|D_{k-2}\right|\right.$ if $\left.i=1\right)$.

3.3. Variation of $\lambda$. The three gap theorem describes the gaps at fixed $\lambda$, while the initial conditions for the survival probability are at all $\lambda>\rho$. So, we need to describe how the variation in $\lambda$ affects the relevant part of the continued fraction expansion, and the length of the relevant part, namely the $(k, i)$ that we need to consider.

Consider the dynamics at fixed $\lambda$. Initially, all gaps are larger than $h$. Best approximation is found for the full convergents, so the first difference to decrease below $h$ must be from a convergent, say $\left|D_{k-1}\right|$ with $k$ now determined by $h$. The second will be in the next sequence of semiconvergents, $\left|D_{k, i}\right|$, possibly the next convergent (if $i=a_{k}$ ). Now, only the largest of the three gaps, namely $\left|D_{k, i-1}\right|$ remains greater than $h$. Finally, the next difference, which is the difference of these two, $\left|D_{k, i+1}\right|$ or $\left|D_{k+1,1}\right|$ will also fall below $h$, leading to complete escape. Thus we have defined a specific $(k, i)$ for each $\lambda$ in a given mushroom (parametrised by $\rho)$.

Now, the condition for a MUPO can be expressed simply in terms of these quantities: If there is a $\lambda=A / B$ such that $0 \leq h<1 / B$ the orbit is periodic and can avoid the hole, so we have a MUPO. In this case, all the differences $D_{k, i}$ are 

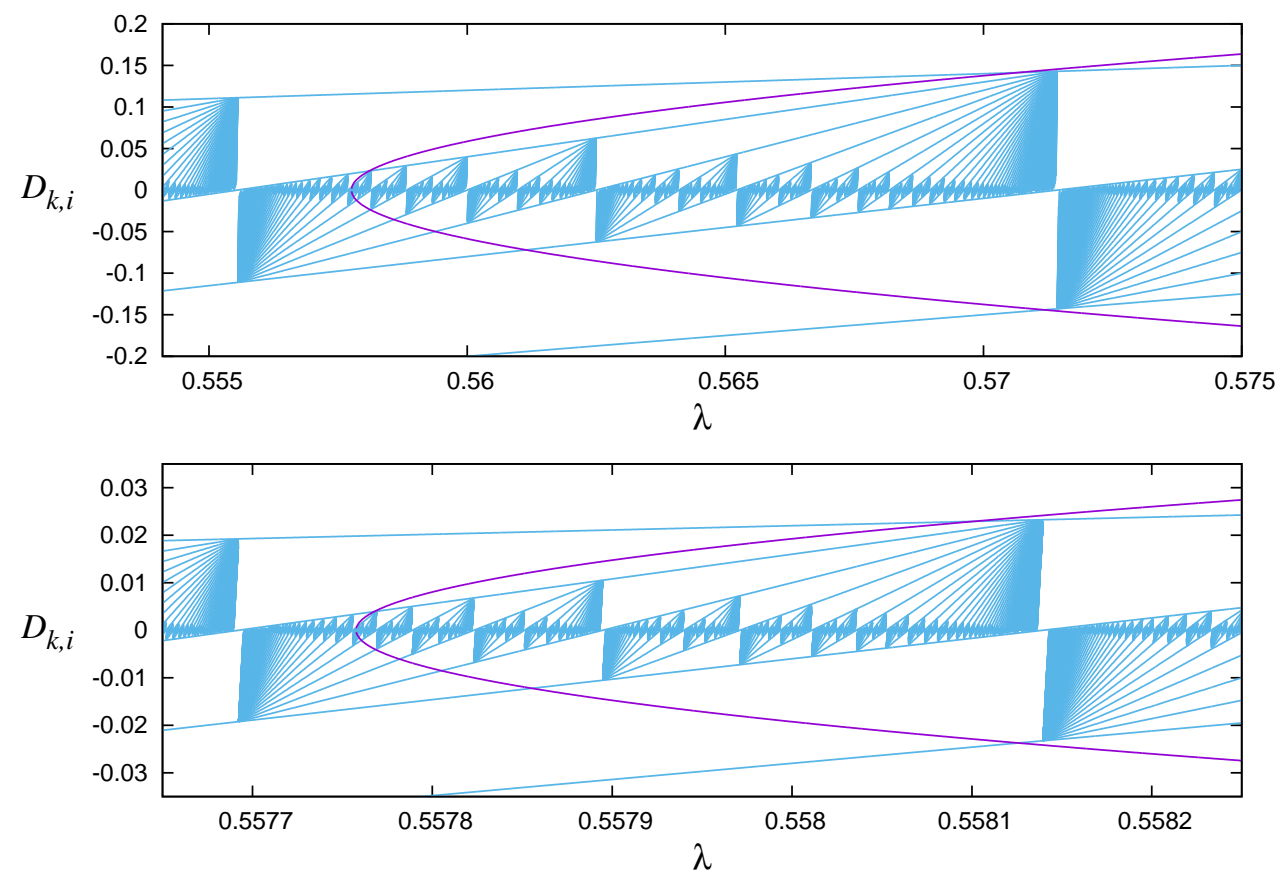

Figure 5. Close-ups of Fig. 4 showing $\pm h$ (the hole size) for the known MUPO-free case $r=\cos \left(\frac{5+\sqrt{2}}{23} \pi\right)$ as the parabolalike curve. Note that this value of $r$ barely avoids a MUPO at $\lambda=4 / 7=0.571 \ldots$, since $h$ is only slightly greater than $1 / 7$ at that point. Also, due to the periodicity of the continued fraction expansion, the behaviour near the intersection with the $x$-axis is self-similar.

multiples of $1 / B$ and so greater than $h$. Conversely, if there is at least one nonzero difference less than $h$ at all $\lambda \geq \rho$, then no MUPO exists. See Fig. 5 .

Transitions in the continued fraction expansion itself take place at rational $\lambda$. However, there is at least one non-zero difference less than $h$, which we can identify as $D_{k-1}$. The next to fall below $h, D_{k, i}$ is a negative multiple of this at the rational point, so also non-zero. Thus none of the quantities needed to define these differences, $k, i$ or $a_{k-1}$ have changed across the transition. Furthermore, as shown in Lemma 5.1 the value of $k$ can only decrease as $\lambda$ increases. Together, these show that variations in the continued fraction expansion in $\lambda$ are never relevant; the survival probability $P(t)$ may be calculated using the (fixed) expansion of $\rho$.

Transitions in $(k, i)$ as $\lambda$ varies must therefore correspond only to cases where $\left|D_{k, i}\right|=h$ and/or $\left|D_{k-1}\right|=h$. Note that

- $D_{k, i}$ and $h$ increase with $\lambda$. 
- $D_{k, i}$ is linear and $h$ concave in $\lambda$.

- $D_{k, i}>0$ iff $k$ is even (see Eq. 3.9p.

The second point shows that $\left|D_{k, i}\right|=h$ can have at most two solutions. Actually, as shown in Lemma 5.1. only the solution where $h$ is increasing faster than $D_{k, i}$ is relevant, since the other possibility leads to the existence of a MUPO.

Definition 3.1. Incipient MUPO. Transition of both types occurring simultaneously, that is, $\left|D_{k, i}\right|=\left|D_{k-1}\right|=h$.

At the relevant $\lambda$, the hole size is exactly the same size as both relevant differences. Increasing $\rho$ by an arbitrarily small amount leads to a MUPO, hence the terminology. At the transition point itself, all orbits at and near this value of $\lambda$ escape in finite time, so there is no effect on the long time survival probability. In order to satisfy the equation at some rational rotation number $A / B$, we have

$$
r=\frac{\cos \frac{\pi}{2} \frac{A}{B}}{\cos \frac{\pi}{2} \frac{1}{B}}
$$

Conjecture 3.2. No incipient MUPOs without MUPOs. The above equation for $r$ is never satisfied without the existence of another (real) MUPO.

The equation for an incipient MUPO has a countable number of solutions, while the condition for the non-existence of a MUPO has (from previous studies) a zero measure set of solutions. Thus, a probabilistic argument suggests there is no overlap. In particular, there is no reason to expect the continued fraction expansion of $\frac{2}{\pi} \arccos r$ to have bounded even partial quotients, which is a necessary condition for being MUPO-free. A numerical search does not find any solutions, although there are examples for which the smallest MUPO is rather long. One of the simplest is $\lambda=\frac{3}{4}, h=\frac{1}{4}, l=\cos \frac{3 \pi}{8}=\frac{\sqrt{2-\sqrt{2}}}{2}, r=\frac{l}{\cos (\pi / 8)}=\sqrt{2}-1$. In this case, the shortest MUPO has $A / B=1181 / 1622$.

3.4. Survival probability at fixed $\lambda$. Now let us calculate the survival probability, that is, the measure of surviving orbits in the presence of the hole of size $h$. For now, the initial conditions have fixed $l$ and hence $\lambda$ and $h$ but are otherwise uniformly distributed round the circle. Recall that the condition for a MUPO is that there is a $\lambda=A / B$ for which $h<1 / B$.

Putting the above pieces together, we find the survival probability as a function of collisions:

$$
P_{\lambda}(N)=\left\{\begin{array}{cc}
1-N h & N \leq B_{k-1} \\
1-B_{k-1} h-\left(N-B_{k-1}\right)\left|D_{k-1}\right| & B_{k-1}<N \leq B_{k, i} \\
\left(B_{k-1}+B_{k, i}-N\right)\left(\left|D_{k, i-1}\right|-h\right) & B_{k, i}<N \leq B_{k-1}+B_{k, i} \\
0 & N>B_{k-1}+B_{k, i}
\end{array}\right.
$$

Note that for moderate $h$, it is quite possible for the most of the particles to remain for a long time if $a_{k}$ is large and hence $i$ and $B_{k, i}$ can be large.

3.5. Integrating over $\lambda$. We place initial conditions uniformly in the semicircular cap of the mushroom with uniform directions; this corresponds to the equilibrium invariant measure of the billiard flow: See for example Ref. [1]. Integrating 
over an arbitrary function of $\lambda$, we have

$$
\begin{aligned}
\int f(\lambda) d \mu & =\int_{0}^{2 \pi} \frac{d \phi}{2 \pi} \int_{0}^{\pi} \frac{d \theta}{\pi} \int_{0}^{1} 2 r d r f\left(\frac{2}{\pi} \arccos |r \sin (\theta-\phi)|\right) \\
& =\int_{0}^{1} f(\lambda) 2 \sin ^{2}\left(\frac{\pi}{2} \lambda\right) d \lambda \\
& =\int f(\lambda) d \mu_{\lambda}
\end{aligned}
$$

giving explicitly the associated measure $d \mu_{\lambda}$ for $\lambda \in(0,1) \subset \mathbb{R}$. Here, $r$ is radial distance, $\theta$ gives the angular position, $\phi$ gives the direction of the particle, hence the angular momentum is $l=|r \sin (\theta-\phi)|$.

We further restrict to the chaotic region, $l<r$ or equivalently $\lambda>\rho$, which requires normalisation by a further constant

$$
c_{\rho}=\int_{\rho}^{1} 2 \sin ^{2}\left(\frac{\pi}{2} \lambda\right) d \lambda=1-\rho+\frac{2}{\pi} \sin \frac{\pi \rho}{2} \cos \frac{\pi \rho}{2}
$$

Thus, the full survival probability is

$$
P(t)=c_{\rho}^{-1} \int P_{\lambda}\left(\left\lfloor\frac{t}{\tau_{\lambda}}\right\rfloor\right) d \mu_{\lambda}
$$

which in principle can be evaluated exactly at fixed $t$, splitting $\lambda$ into regions with differing $N=\left\lfloor t / \tau_{\lambda}\right\rfloor$ (if applicable), and into the different regimes of Eq. (3.11), and noting the integrals

$$
\begin{aligned}
\int d \mu_{\lambda}= & \lambda-\frac{2}{\pi} l \sqrt{1-l^{2}}+C \\
\int h d \mu_{\lambda}= & -\int\left(\frac{2}{\pi} \arccos \frac{l}{r}\right)\left(\frac{4}{\pi} \sqrt{1-l^{2}}\right) d l \\
= & \frac{2}{\pi^{2}}\left[\operatorname{Li}_{2}\left(r^{-1} e^{i \pi(\lambda+h) / 2}\right)+\operatorname{Li}_{2}\left(r^{-1} e^{-i \pi(\lambda+h) / 2}\right)\right. \\
& -\frac{\pi^{2}}{4}\left(h^{2}-2 h-2 \lambda\right)-\pi l h \sqrt{1-l^{2}} \\
& \left.+\left(1-r^{2}\right) \ln \left(\sqrt{1-l^{2}}+\sqrt{r^{2}-l^{2}}\right)+\sqrt{1-l^{2}} \sqrt{r^{2}-l^{2}}\right]+C
\end{aligned}
$$

where $\mathrm{Li}_{2}$ is the dilogarithm and $h$ and $l$ are the usual functions of $\lambda$. We do not need the explicit form of $P(t)$ for any of our results, however.

\section{Proofs of the theorems}

Sec. 4.1 shows that the positive finite limits in Thm. 1.1. Sec. 4.2 uses the almost piecewise linearity of $P(t)$ to get the explicit bound, completing the proof of Thm. 1.1. Sec. 4.3 contains the proof of Thm. 1.2 .

4.1. Bounds on the survival probability. From this point we assume that in addition to the MUPO-free condition, the partial quotients of $\rho$ are bounded. Possible effects of violating this condition were discussed briefly in the introduction. We have Lemma 5.2 which considers the interval of relevant $\lambda$ for large $t$. In particular, there is a function $\lambda_{\max }(t)$ satisfying

$$
\lambda_{\max }(t)=\rho+\mathcal{O}\left(t^{-2}\right)
$$


at large $t$ so that $P_{\lambda}\left(\left\lfloor\frac{t}{\tau_{\lambda}}\right\rfloor\right)=0$ for $\lambda>\lambda_{\max }(t)$.

The integral for $P(t)$ directly gives an upper bound

$$
P(t)<\frac{C_{\max }}{t^{2}}
$$

To get the equivalent lower bound

$$
P(t)>\frac{C_{\min }}{t^{2}}
$$

at sufficiently large $t$ we note that $P_{\lambda}(N) \geq 1-N h$ for all $N$ and integrate until this vanishes, a region of order $t^{-2}$. This completes the proof of positive and finite limits in Theorem 1.1.

4.2. Approximate piecewise linearity. We see from Eq. 3.11 that $P_{\lambda}(N)$ is a piecewise linear function of $N$. We would like to see whether this applies also to $P(t)$, which we now approximate. It is easy to see that Eq. 4.1 implies

$$
\frac{t}{\tau_{\rho}}-\frac{t}{\tau_{\lambda_{\max }}}=\mathcal{O}\left(t^{-1}\right)
$$

that is, there are at most two relevant values of $N$ at sufficiently long times. From Eq. 3.11, $0<P_{\lambda}(N)<1$ and $P_{\lambda}(N+1)-P_{\lambda}(N) \leq h$, where $h=\mathcal{O}\left(t^{-1}\right)$. Putting this together we see that

$$
|P(t)-\tilde{P}(t)|=\mathcal{O}\left(t^{-3}\right)
$$

where the approximated integral

$$
\tilde{P}(t)=c_{\rho}^{-1} \int P_{\rho}\left(\left\lfloor\frac{t}{\tau_{\rho}}\right\rfloor\right) d \mu_{\lambda}
$$

is piecewise linear with transitions at $\tau_{\rho} B_{k, i}$. This is observed numerically in Fig. 6 .

Furthermore, infinitely many transition points are spaced with ratios greater than the golden ratio, as shown in Lemma 5.3 , and a piecewise linear function such as $\tilde{P}(t)$ with asymptotic form $t^{-2}$ and transition points with such spacing must have a limiting ratio of at least $\frac{32}{27}$ as shown in Lemma 5.4. From above, $|\tilde{P}(t)-P(t)|$ is at most of order $t^{-3}$ and so negligible in the limit, so the result holds for $P(t)$ as well. This complete the proof of Theorem 1.1 .

4.3. Eventually periodic continued fractions. In this final section we prove Theorem 1.2, for which the continued fraction of $\rho$ is eventually periodic. As shown in standard texts including Ref. $\mathbf{1 9}$, this condition is exactly that $\rho$ is a quadratic irrational.

We can write the recurrence relation Eq. 3.7 as

$$
v_{k}=A_{k} v_{k-1}
$$

with

$$
\begin{aligned}
v_{k} & =\left(\begin{array}{c}
B_{k} \\
B_{k-1}
\end{array}\right) \\
A_{k} & =\left(\begin{array}{cc}
a_{k} & 1 \\
1 & 0
\end{array}\right)
\end{aligned}
$$

so that $\operatorname{det} A_{k}=-1$. Also, any product of $A$ matrices has only positive entries, so by the Perron-Frobenius theorem it has a simple positive eigenvalue of strictly maximal magnitude. Let $p$ be the period of the continued fraction expansion of 


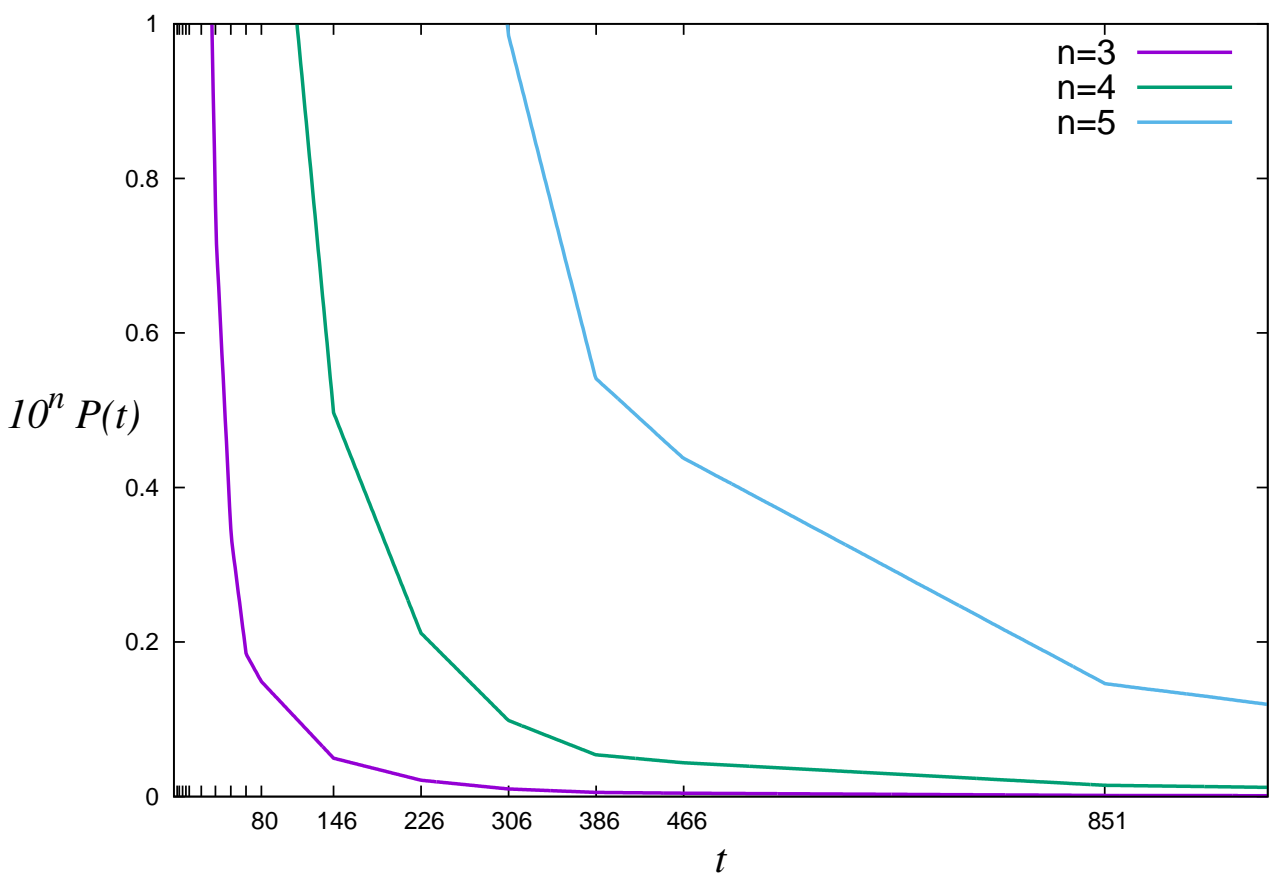

FiguRE 6 . The survival probability is approximately piecewise linear, with pieces defined by $\tau_{\rho} B_{k, i}$, here represented on the axis by the nearest integer. As above, we choose $r=\cos \left(\frac{5+\sqrt{2}}{23} \pi\right)$.

$\rho$. All products of $2 p$ consecutive $A$ matrices in the periodic part of the expansion have unit determinant. They are also cyclic permutations and hence have the same eigenvalues $\beta>\beta^{-1}>0$. Since $\beta$ and $\beta^{-1}$ are the roots of a monic quadratic polynomial with integer coefficients they are conjugate quadratic irrationals.

Now let $k$ be in the periodic part of the expansion (that is, sufficiently large, and fixed). We have

$$
B_{k}=C_{k} \beta^{\frac{k}{2 p}}+\mathcal{O}\left(\beta^{-\frac{k}{2 p}}\right)
$$

where $C_{k+2 p}=C_{k}$. Noting Eq. 3.9 and the periodicity of $\zeta_{k}$, we find

$$
D_{k}=\tilde{C}_{k} \beta^{-\frac{k}{2 p}}+\mathcal{O}\left(\beta^{-3 \frac{k}{2 p}}\right)
$$

where $\tilde{C}_{k+2 p}=\tilde{C}_{k}$, and equations with related periodic constants for $B_{k, i}$ and $D_{k, i}$. This self-similarity of the differences can be observed in Fig. 5 above.

We now define $t^{\prime}=\beta^{m} t$ for some large integer $m$ and large $t$, and want to compare $\tilde{P}\left(t^{\prime}\right)$ with $\tilde{P}(t)$. We see that the various quantities scale: $N^{\prime}=t^{\prime} / \tau_{\rho}=$ $\beta^{m} N$. For most $t$, we have $\left(k^{\prime}, i^{\prime}\right)=(k+2 m p, i)$, so we consider $D_{k+2 p m, i}=$ $\beta^{-m} D_{k, i}\left(1+\mathcal{O}\left(\beta^{-\frac{k}{p}}\right)\right)$, equating this with $h$ to find the transitions in $\lambda$, we have, 
using Eq. 3.2. $\lambda^{\prime}-\rho=\beta^{-2 m}(\lambda-\rho)\left(1+\mathcal{O}\left(\beta^{-\frac{k}{p}}\right)\right)$. Thus when $\left(k^{\prime}, i^{\prime}\right)=(k+2 m p, i)$,

$$
\left|\beta^{2 m} t^{2} \tilde{P}\left(\beta^{m} t\right)-t^{2} \tilde{P}(t)\right|=\mathcal{O}\left(\beta^{-\frac{2 k}{p}}\right)
$$

The nonsmooth points of $\tilde{P}(t)$ do not exactly scale: From above we have $\tau_{\rho} B_{k+2 m p, i}=$ $\beta^{m} \tau_{\rho} B_{k, i}\left(1+\mathcal{O}\left(\beta^{-\frac{k}{p}}\right)\right)$. This means that for $t$ close to a transition point, it is possible that $\left(k^{\prime}, i^{\prime}\right) \neq(k+2 m p, i)$. In this case we consider a value $\tilde{t}$ close to $t$ but across the transition. We have $|\tilde{t}-t|=\mathcal{O}\left(\beta^{-\frac{k}{2 p}}\right)$. Also, $t^{2} \tilde{P}(t)$ has positive upper and lower bounds and $\tilde{P}(t)$ is convex, implying that the variation satisfies

$$
\left|\frac{\tilde{P}(\tilde{t})-\tilde{P}(t)}{\tilde{t}-t}\right|<\frac{C}{\min (\tilde{t}, t)^{3}}
$$

so that Eq. 4.12 is still satisfied. Finally we use $\tilde{P}(t)$ to approximate $P(t)$ and take $m \rightarrow \infty$ then $k \rightarrow \infty$ to obtain the result of Theorem 1.2 .

\section{Lemmas and their proofs}

Lemma 5.1. Monotonicity of transitions. A difference becomes relevant only as $\lambda$ increases. Precisely, for a MUPO-free $r$, if there is some $(k, i)$ and $\lambda$ for which $f(\lambda)=h-\left|D_{k, i}\right|=0$, then $f^{\prime}(\lambda)>0$ at that point.

PROOF. If $k$ is odd, then $D_{k, i}<0$, so both terms in $f^{\prime}(\lambda)$ are positive and we are finished.

In the case $k$ is even, assume that the assertion is false. Then $D_{k, i}$ continues to higher $\lambda$ until it terminates at $\lambda=A_{k-1} / B_{k-1}$ at which it is equal to $1 / B_{k-1}$ using the equations in Sec. 3.2. Since $D_{k, i}$ is a linear function of $\lambda$ and $h$ is concave, $f^{\prime}(\lambda)$ remains negative, hence $f\left(A_{k-1} / B_{k-1}\right)<0$. This means that $0<h<1 / B_{k-1}$ at this point, which would imply that there is a MUPO with rotation number $A_{k-1} / B_{k-1}$, a contradiction.

Lemma 5.2. Size of $\lambda$ interval. For MUPO-free $\rho$ with bounded partial quotients, $P_{\lambda}\left(\left\lfloor\frac{t}{\tau_{\lambda}}\right\rfloor\right)=0$ for $\lambda>\lambda_{\max }(t)$ where

$$
\lambda_{\text {max }}(t)=\rho+\mathcal{O}\left(t^{-2}\right)
$$

as $t \rightarrow \infty$.

Proof. Fix a time $t>0$ and constant $0<C_{1}<t^{2}(1-\rho)$. If

$$
\lambda>\rho+\frac{C_{1}}{t^{2}}
$$

we have

$$
h>\frac{C_{2}}{t}
$$

using the expansion of $h$, Eq. 3.2. Here, $C_{2}$ is a constant proportional to $C_{1}$. The value of $k$ is determined so that $\left|D_{k-1}\right|$ is the first difference to fall below $h$. In particular

$$
\left|D_{k-2}\right|>h
$$

Equation 1.4.5 of Ref $\mathbf{1 9}$ gives

$$
\left|D_{k-2}\right| \leq \frac{1}{B_{k-1}}
$$


Thus we have

$$
B_{k-1}<\frac{t}{C_{2}}
$$

Using the recurrence relation for the $B_{k}$, the boundedness of the $a_{k}$ (because there are no MUPOs we may use the continued fraction expansion of $\rho$ rather than $\lambda$ ) we have

$$
B_{k-1}+B_{k, i}<\frac{t}{C_{3}}
$$

with a constant $C_{3}$ proportional to $C_{2}$. We also have

$$
N=\left\lfloor\frac{t}{\tau_{\lambda}}\right\rfloor>\frac{t}{2}-1>\frac{t}{3}
$$

since $\tau_{\lambda}<2$ for all $\lambda$ and $t$ can be chosen arbitrarily large. If we choose $t$ large enough, we can choose a large enough $C_{1}<t^{2}(1-\rho)$ so that $C_{3}>3$ and we find from Eq. (3.11) that $P_{\lambda}(N)=0$ as required.

Lemma 5.3. Ratio of semiconvergent denominators. For any $\lambda \notin \mathbb{Q}$, its (infinite) sequence of semiconvergent denominators $B_{k, i}$ has ratio of successive terms at least $g=\frac{1+\sqrt{5}}{2}$ infinitely often.

Proof. We have

$$
\frac{B_{k, 1}}{B_{k-1}}=\frac{B_{k-1}+B_{k-2}}{B_{k-1}}=1+\frac{B_{k-2}}{B_{k-1}}=\left[1 ; a_{k-1}, \ldots, a_{1}\right]
$$

where the final equality comes from Ref. [19, section 1.6.

If $\lambda \sim g$, that is, the $a_{k}$ have a tail consisting only of $1 \mathrm{~s}$, we have $B_{k-2} / B_{k-1} \rightarrow$ $g^{-1}$ alternating above and below this value, and we are finished.

If there are are infinitely many $1 \mathrm{~s}$ in the partial quotients, any $k$ for which $a_{k-1}=1$ and $a_{k-2}>1$ will have $\left[1 ; a_{k-1}, a_{k-2} \ldots, a_{1}\right]>5 / 3>g$ and we are likewise finished.

If all but a finite number of partial quotients are greater than 1 , instead consider (for both $a_{k}>1$ and $a_{k-1}>1$ )

$$
\frac{B_{k, 2}}{B_{k, 1}}=\frac{2 B_{k-1}+B_{k-2}}{B_{k-1}+B_{k+2}}=1+\frac{1}{1+\frac{B_{k-2}}{B_{k-1}}}=\left[1 ; 1, a_{k-1}, a_{k-2}, \ldots a_{1}\right]>\frac{5}{3}>g
$$

Lemma 5.4. Minimum variation of piecewise linear functions. Let $f: \mathbb{R}^{+} \rightarrow$ $\mathbb{R}^{+}$be piecewise linear with consecutive transition points $t_{i}$ having ratio at least the golden ratio infinitely often. If the limits are positive and finite as $t \rightarrow \infty$ then

$$
\frac{\limsup t^{2} f(t)}{\liminf t^{2} f(t)} \geq \frac{32}{27} \approx 1.185 \ldots
$$

Proof. According to the assumptions we can find infinitely many consecutive transition points (two of which denoted $t_{1}, t_{2}$ ) such that $t_{2} / t_{1} \geq g$. Within such an interval, writing $t_{2} / t_{1}=G$,

$$
t^{2} f(t)=t^{2}\left[\beta-\gamma\left(t-t_{1}\right)\right]
$$

where

$$
\beta=f\left(t_{1}\right)>0, \quad \gamma=-\frac{f\left(G t_{1}\right)-f\left(t_{1}\right)}{(G-1) t_{1}}>0
$$


are constant. Possible supremum/infimum points consist of the left and right endpoints, and a turning point:

$$
\begin{aligned}
L & =t_{1}^{2} f\left(t_{1}\right)=\beta t_{1}^{2} \\
T & =t_{c}^{2} f\left(t_{c}\right)=\beta t_{1}^{2} \frac{4}{27 z}(1+z)^{3}, \quad \text { if } t_{c}=t_{1} \frac{2}{3}(1+z) \in\left(t_{1}, G t_{1}\right) \\
R & =t_{2}^{2} f\left(t_{2}\right)=\beta t_{1}^{2} G^{2}\left[1-\frac{G-1}{z}\right]
\end{aligned}
$$

where $z=\beta /\left(\gamma t_{1}\right)$. The turning point is relevant if

$$
\frac{1}{2}<z<\frac{3 G}{2}-1
$$

First we turn to cases where it is not relevant. For $z<1 / 2$ we find, using $G \geq g$, that $R<0$, which is impossible. For $z>3 G / 2-1$ we compute

$$
\frac{R}{L}=G^{2}\left[1-\frac{G-1}{z}\right]>G^{2}\left[1-\frac{G-1}{3 G / 2-1}\right]=\frac{G^{3}}{3 G-2}
$$

The derivative of the right hand side is positive for $G \geq g$. Thus we conclude

$$
\frac{R}{L} \geq \frac{g^{3}}{3 g-2}>\frac{32}{27}
$$

as required. This completes the cases where the turning point is not relevant.

When the turning point is relevant, we have two cases. When $z \geq \frac{G^{2}}{G+1}$ we have $R \geq L$, and so the relevant ratio is

$$
\frac{T}{L}=\frac{4}{27} \frac{(1+z)^{3}}{z} \geq \frac{32}{27}
$$

since we know that $z \geq \frac{G^{2}}{G+1} \geq \frac{g^{2}}{g+1}=1$. Conversely, when $z \leq \frac{G^{2}}{G+1}$, the relevant ratio is

$$
\frac{T}{R}=\frac{4}{27} \frac{(1+z)^{3}}{G^{2}(z+1-G)}
$$

Differentiating (and keeping $G$ constant) we have

$$
\frac{d}{d z} \frac{T}{R}=\left(\frac{z+1}{G(z+1-G)}\right)^{2}(2(z+1)-3 G)<0
$$

since $z<3 G / 2-1$ in order for $T$ to be relevant, as above. Thus its minimum value is obtained at $z=\frac{G^{2}}{G+1}$ :

$$
\frac{T}{R} \geq \frac{4}{27} \frac{\left(1+\frac{G^{2}}{G+1}\right)^{3}}{G^{2}\left(\frac{G^{2}}{G+1}+1-G\right)}=\frac{4}{27} \frac{\left(G^{2}+G+1\right)^{3}}{G^{2}(G+1)^{2}}
$$

This function is increasing for $G \geq g$, thus we find

$$
\frac{T}{R} \geq \frac{4}{27} \frac{\left(g^{2}+g+1\right)^{3}}{g^{2}(g+1)^{2}}=\frac{32}{27}
$$

as required. 


\section{References}

1. E G Altmann, Intermittent chaos in Hamiltonian dynamical systems, Ph.D. thesis, Wuppertal, 2007.

2. Eduardo G Altmann, Adilson E Motter, and Holger Kantz, Stickiness in mushroom billiards, Chaos 15 (2005), no. 3, 033105.

3. Eduardo Goldani Altmann, T Friedrich, AE Motter, H Kantz, and A Richter, Prevalence of marginally unstable periodic orbits in chaotic billiards, Phys. Rev. E 77 (2008), no. 1, 016205.

4. Or Alus, Shmuel Fishman, and James D. Meiss, Probing the statistics of transport in the Hénon map, arxiv:1512.04723 (2015).

5. A Bäcker, R Ketzmerick, S Löck, Marko Robnik, Gregor Vidmar, Ruven Höhmann, Ulrich Kuhl, and H-J Stöckmann, Dynamical tunneling in mushroom billiards, Phys. Rev. Lett. 100 (2008), no. 17, 174103.

6. Alex H Barnett and Timo Betcke, Quantum mushroom billiards, Chaos 17 (2007), no. 4, 043125 .

7. LA Bunimovich, On billiards close to dispersing, Sbornik: Mathematics 23 (1974), no. 1, 45-67.

8. Leonid A Bunimovich, Mushrooms and other billiards with divided phase space, Chaos 11 (2001), no. 4, 802-808.

9. __ Fine structure of sticky sets in mushroom billiards, J. Stat. Phys. 154 (2014), no. 1-2, 421-431.

10. Leonid A Bunimovich and Luz V Vela-Arevalo, Many faces of stickiness in Hamiltonian systems, Chaos 22 (2012), no. 2, 026103.

11. N. I. Chernov and R Markarian, Chaotic billiards, Amer. Math. Soc., Providence, 2006.

12. Giampaolo Cristadoro and Roland Ketzmerick, Universality of algebraic decays in Hamiltonian systems, Phys. Rev. Lett. 100 (2008), no. 18, 184101.

13. C Dembowski, H-D Gräf, A Heine, R Hofferbert, H Rehfeld, and A Richter, First experimental evidence for chaos-assisted tunneling in a microwave annular billiard, Phys. Rev. Lett. 84 (2000), no. 5, 867 .

14. Carl Dettmann, Open circle maps: small hole asymptotics, Nonlinearity 26 (2013), no. 1, 307.

15. Carl P Dettmann and Orestis Georgiou, Open mushrooms: stickiness revisited, J. Phys. A 44 (2011), no. 19, 195102.

16. Barbara Dietz, T Friedrich, M Miski-Oglu, A Richter, and F Schäfer, Spectral properties of Bunimovich mushroom billiards, Phys. Rev. E 75 (2007), no. 3, 035203.

17. Sean Gomes, Percival's conjecture for the Bunimovich mushroom billiard, arXiv:1504.07332 (2015).

18. Alastair Robertson, Stickiness in MUPO-free mushroom billiards, Undergraduate project, University of Bristol (2013).

19. Andrew M Rockett and Peter Szüsz, Continued fractions, vol. 47, World Scientific, 1992.

20. Noel B Slater, Gaps and steps for the sequence $n \theta \bmod 1$, Mathematical Proceedings of the Cambridge Philosophical Society, vol. 63, Cambridge Univ Press, 1967, pp. 1115-1123.

21. Vera T Sós, On the distribution mod 1 of the sequence n $\alpha$, Ann. Univ. Sci. Budapest, Eötvös Sect. Math 1 (1958), 127-134.

22. Sergei Tabachnikov, Geometry and billiards, Amer. Math. Soc., Providence, 2005.

23. Satoru Tsugawa and Yoji Aizawa, Stagnant motion in Hamiltonian dynamics - Mushroom billiard case with smooth outermost KAM tori -, J. Phys. Soc. Japan 83 (2014), no. 2, 024002 .

24. Tony Van Ravenstein, The three gap theorem (Steinhaus conjecture), J. Austral. Math. Soc.(Series A) 45 (1988), 360-370.

25. Lei Ying, Guanglei Wang, Liang Huang, and Ying-Cheng Lai, Quantum chaotic tunneling in graphene systems with electron-electron interactions, Phys. Rev. B 90 (2014), no. 22, 224301.

School of Mathematics, University of Bristol, Bristol BS8 1TW, UK

E-mail address: Carl.Dettmann@bristol.ac.uk 